Classification

Physics Abstracts

$61.40-61.50 \mathrm{~K}-64.70 \mathrm{~K}$

\title{
Local molecular order in the glassy crystalline phase of cyanoadamantane : diffuse $X$-ray scattering analysis
}

\author{
M. Descamps, C. Caucheteux, G. Odou and J. L. Sauvajol \\ Laboratoire de Dynamique des Cristaux moléculaires (*), Université de Lille I, \\ 59655 Villeneuve d'Ascq Cedex, France
}

(Reçu le 21 février 1984, accepté le 30 mai 1984)

\begin{abstract}
Résumé. - Dans cette Lettre nous présentons les résultats d'expériences de diffusion de rayons $\mathbf{X}$ effectuées sur le cyanoadamantane en phase plastique (I) et en phase « cristal vitreux » (Ig) obtenue par trempe de la phase(I). L'aspect général des figures de diffusion est dans ce dernier cas plus structuré. De plus, il apparaît en phase vitreuse, des taches diffuses fines aux points $\mathrm{X}$ des zones de Brillouin. Nous proposons une interprétation en termes de domaines antiferroélectriques engrainés. La longueur de corrélation correspondante serait alors d'environ $20 \AA$.
\end{abstract}

\begin{abstract}
This Letter presents the results of X-ray scattering experiments performed on cyanoadamantane in its plastic phase (I) and its " glassy crystal » state (Ig) obtained by the quenching of phase (I). The patterns associated with this latter phase are more structured and are characterized by the presence of narrow spots at the $X$ boundary points. An interpretation is proposed in terms of geared antiferroelectric domains. The coherence length is $\xi \simeq 20 \AA$.
\end{abstract}

\section{Introduction.}

In this Letter we report the first results of X-ray scattering experiments performed on the plastic phase and the " glassy crystal » phase of cyanoadamantane (CN-ADM). This latter state has been recently discovered [1] and its main properties are summarized below.

At room temperature, CN-ADM (Fig. 1) has an orientational disordered phase (I) with a facecentred cubic structure $\operatorname{Fm} 3 \mathrm{~m}(Z=4)$. The dipolar axis can randomly take six orientations very closely located to the $\langle 001\rangle$ directions [2]. The corresponding reorientations are slow with correlation times of about $\tau_{\mathrm{c}} \sim 3 \times 10^{-7}$ s. Reorientation processes have also been detected in a higher frequency range $\left(\tau_{\mathrm{u}} \sim 3.5 \times 10^{-12} \mathrm{~s}\right)$ where they correspond to fast uniaxial rotations around the dipolar axis [3]. When grown at temperatures below $T \sim 380 \mathrm{~K}$, the crystal adopts a monoclinic ordered phase (II), $\mathrm{C}^{2} / \mathrm{m}[4]$; in this phase, the dipolar axes are situated in the (a, c) plane and arrange themselves in an antiferroelectric order. Due to a very easy undercooling, it is difficult to reach phase II by cooling phase I; with cooling rates of a few degrees/minute or more, the transition $(\mathrm{I} \rightarrow \mathrm{II})$ is suppressed. If the sample is rapidly cooled to a low enough temperature,

(*) ERA, CNRS 465. 


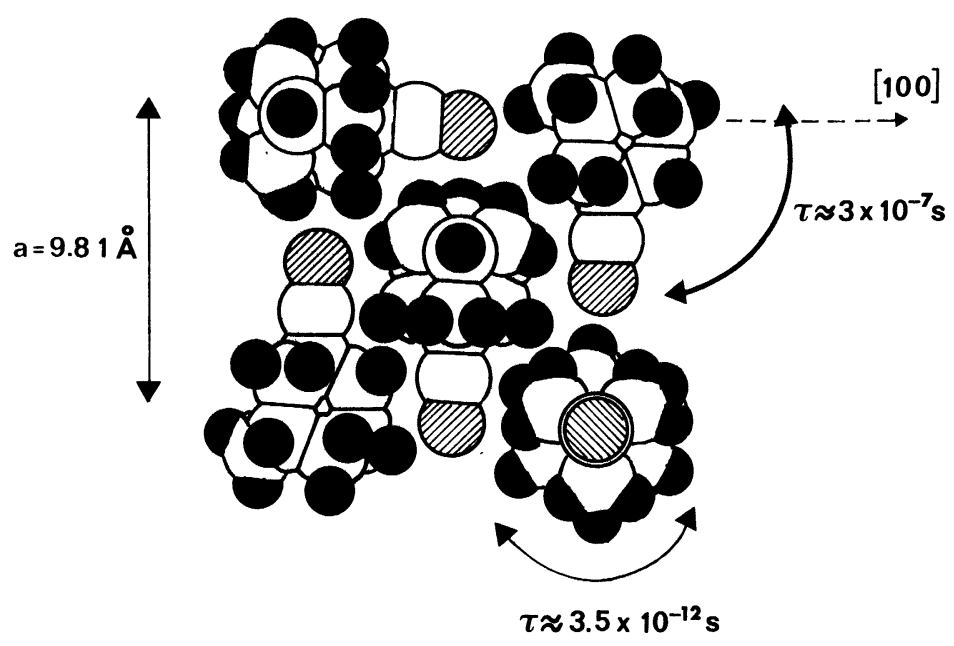

Fig. 1. - A possible instantaneous configuration of the CN-ADM molecules in the orientationally disordered phase (I). The two types of rotations are indicated. In the glassy phase, the dipolar tumbling $\left(\tau_{\mathrm{c}}\right)$ is frozen in.

the reheating behaviour shows all the thermodynamic characteristics of a glassy transition at $T_{\mathrm{g}}$ [1]. Depending on the cooling rate the succession of « phases » is the following :

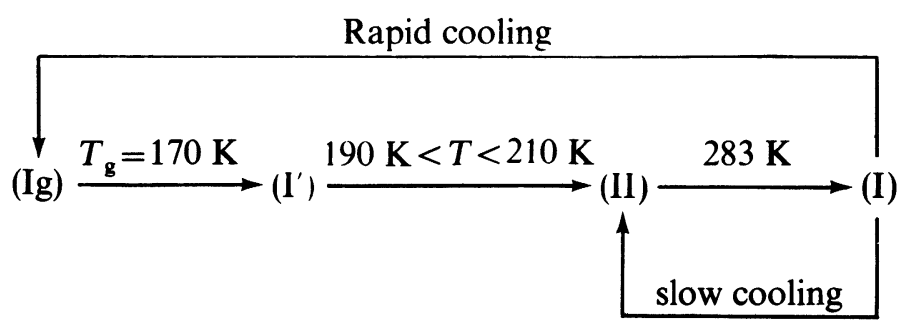

(Ig) and $\left(\mathrm{I}^{\prime}\right)$ respectively label the glassy " phase " and the undercooled metastable form of (I). (Ig) is thus a so-called " glassy-crystal phase " [5]. Up to now this phenomenon has been mainly studied for the particular case of cyclohexanol $[5,6]$. Recent experimental results on CN-ADM have contributed to the microscopic understanding of this intriguing state : single crystal diffraction experiments on the glassy " phase " establish that the translational order of the plastic phase is preserved as well as the time average of both types of orientational disorder [7]. Low frequency dielectric relaxation experiments [8] clearly show that the dipolar tumbling is frozen in at $T_{\mathbf{g}}$ and that the corresponding critical absorption frequency obeys a Vögel-Fülcher law. On the other hand NMR measurements show that the uniaxial reorientations remain in (Ig) [3].

As the average long range order is unchanged, we examine here if the glassy state exhibits a modification of the short range molecular order. Since this particular state has crystalline properties and the glassy aspect seems to be related only to the molecular orientations, we hope to obtain more conclusive results than with the usual glasses obtained by quenching a liquid. 


\section{Experimental.}

Smail single crystals of phase (I) have been obtained by slow evaporation of a saturated solution of purified CN-ADM in methanol. In order to get an extended view of the scattering we used the classical X-ray Laüe technique (Mo incident radiation). The diffuse scattering was recorded on a fixed photographic film located at $85 \mathrm{~mm}$ from the sample. The more interesting $\mathbf{Q}$ zones selected for detailed study have been quantitatively examined using an automatic X-ray diffractometer (monochromatic incident wavelength $\mathrm{MoK} \alpha: 0.711 \AA$ ).

The single crystals of phase (I) could be quenched easily depending on the quality of the core and the surface of the crystal. In the Laüe experiments the crystal was quenched rapidly by placing it in a gaseous nitrogen flow at $113 \mathrm{~K}$. In the diffractometer experiments the glassy phase was obtained by quenching the crystal to $113 \mathrm{~K}$ in five minutes. Up to now the most detailed studies have been done at two temperatures $293 \mathrm{~K}$ (I) and $113 \mathrm{~K}$ (lg).

\section{The $\mathrm{X}$-ray diagrams.}

The features of the (I) patterns are shown on figures $2 \mathrm{a}$ and $3 \mathrm{a}$. The diffuse pattern appears in the form of intense lobes of scattering lying along the $[100]^{*}$ directions with a maximum near 4.700.

a)

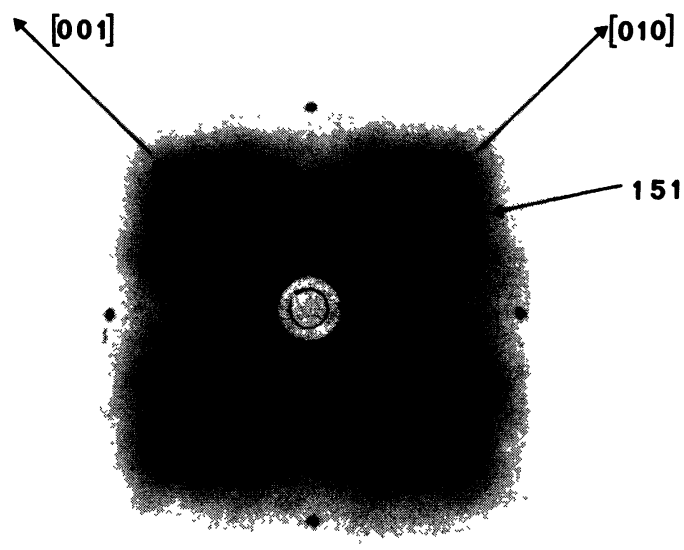

b)

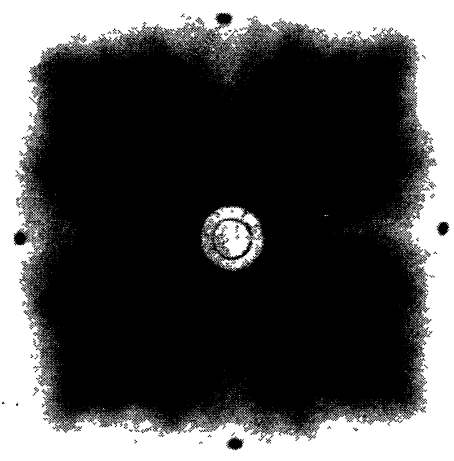

Fig. 2. - Diffuse X-ray scattering (Mo polychromatic Laüe pattern) from the same starting CN-ADM single crystal. Incident beam along a [100] axis. (a) plastic phase (I) at $293 \mathrm{~K}$; (b) glassy crystal state (Ig) at $113 \mathrm{~K}$. 
In contrast, the scattering is very weak in the vicinity of the two fold axis. As has been shown by static neutron scattering [9], this diffuse scattering is mainly due to the orientational disorder of the dipolar axis. The particular localization is to be related to the modulation of the orientational probability : the strong steric repulsions which hinder the reorientations induce a crystalline field with deep wells in the $\langle 100\rangle$ directions. Moreover, when the molecules are in the $\langle 100\rangle$ directions, it frequently happens that some relative configurations are entirely excluded because of steric hindrance. The resulting intermolecular correlations induce the modulation of the scattering actually observed inside the characteristic lobes.

In figures $2 \mathrm{~b}$ and $3 \mathrm{~b}$, patterns of the glassy crystal phase $(\mathrm{Ig})$ are shown for different orientations. All experiments were performed on the same starting crystal. The outlook does not change very much because the average structures of the two phases are nearly the same : both types of orientational disorders remain in the glassy phase and the time averaged orientational probability $P(\Omega)$ is almost unchanged. The main difference occurs in the dynamics which can not affect the $\mathrm{X}$-ray pattern. But we can notice an increase of the contrast; the characteristic lobes of the phase diagrams now appear as the coupling of radial lines. To examine the detailed variation of the diffuse intensity, scans were made with the diffractometer along lines parallel to $\langle 011\rangle$ passing through the reciprocal point 4.700 . This is a region of maximum intensity which is not severely

a)

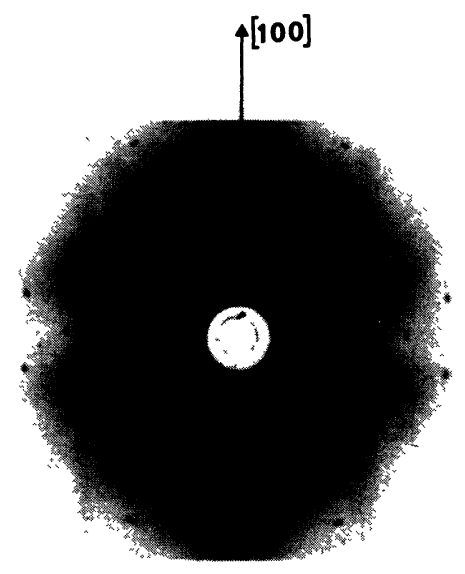

b)

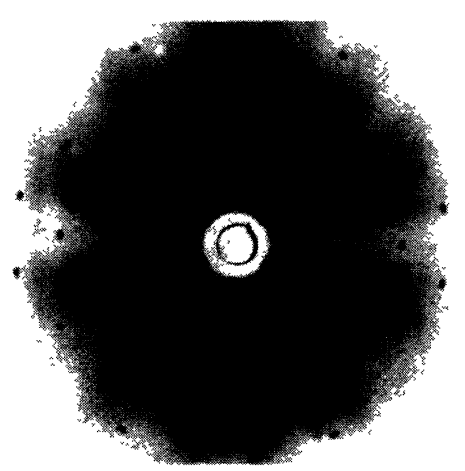

Fig. 3. - Diffuse X-ray scattering (Mo polychromatic Laüe pattern) from the same starting CN-ADM single crystal. Incident beam along a [110] axis. (a) plastic phase (I) at $293 \mathrm{~K}$. (b) glassy crystal state (Ig) at $113 \mathrm{~K}$. 
affected by the thermal scattering (Fig. 4). Experiments at $293 \mathrm{~K}$ (I) and $113 \mathrm{~K}$ (Ig) show a systematic decrease of the scattered intensity which obviously accounts for the sharpening observed on the Laüe photographs. Such a drop has also been observed by Ceccaldi et al. [6] for the cyclohexanol case. As the average orientational structure is unchanged, it seems that we can attribute this evolution to a modification of the local order characterized by the joint probabilities $P\left(\Omega_{i}, \Omega_{j}\right)$.

\section{Local order in the glassy phase.}

In systematic diffractometer scannings of the phases, we have found, in the glassy phase, diffuse scattering spots localized around forbidden reflections $(h+k=2 n+1, k+1=2 n+1$, $h+1=2 n+1)$ at the zone boundary $\mathrm{X}$ points. Such spots are not revealed by the same scans at room temperature (I) but a careful examination shows the existence of very weak increases situated around the $X$ points (Fig. 5a).

The angular limit we have imposed $\left(2 \theta<12^{\circ}\right)$ revealed 11 spots in one height of the space (Table I). We did not find any spots along the twofold axis. A $\mathbf{Q}$ scan along the fourfold axis revealed very weak spots at 100 and 500 ; but we did not find a maximum at 300 . Recently, neutron scattering experiments have confirmed the presence of spots of this kind at greater Bragg angles and have shown that the scattering is quasi elastic [10]. Figure 5a shows a typical scan along the [ $h$ 21]* direction which displays three diffuse spots in $h=0,1$ and 2 ; they correspond to $\mathrm{X}$ points of the B.Z. respectively centred on 200, 111 and 220. The maxima of the scanned spots are all located at less that $0.05 a^{*}$ from the $\mathrm{X}$ point. Figure $5 \mathrm{~b}$ gives particulars of the 021 spot; $\mathbf{Q}$ scans

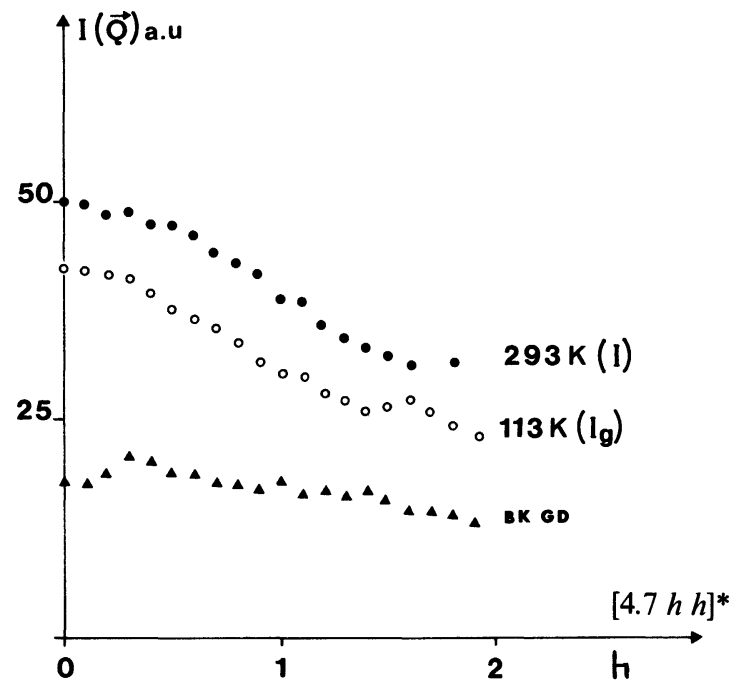

Fig. 4. - $\mathbf{Q}$ scans along $[4.7 h h]^{*}$ on the same starting crystal, in the plastic and in the glassy state.

Table I. - Localization of the spots observed at X boundary points.

$\mathrm{X}$ point coordinates

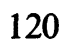

012

121

122
201

102

112
Coordinate of the B.Z. centre

200 and equivalents 
a)

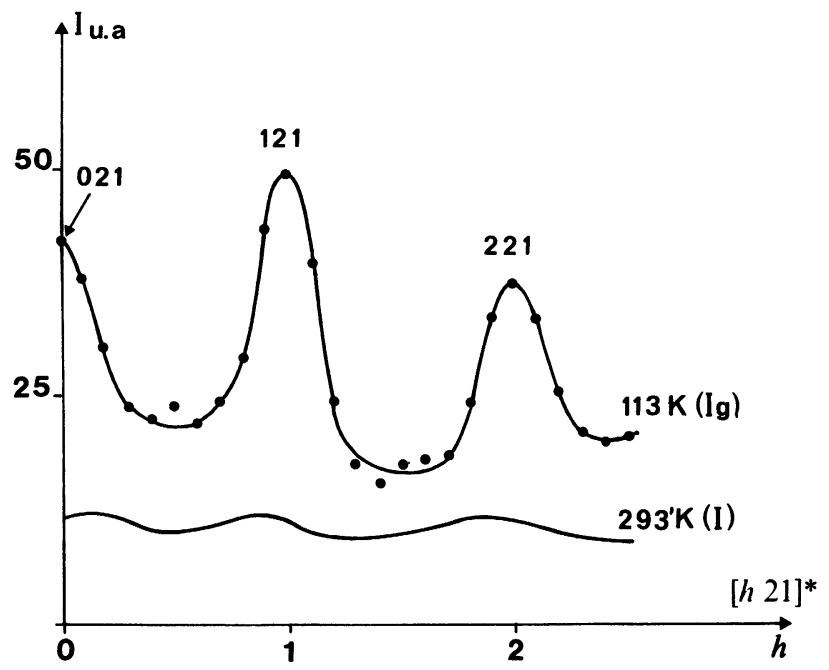

b)
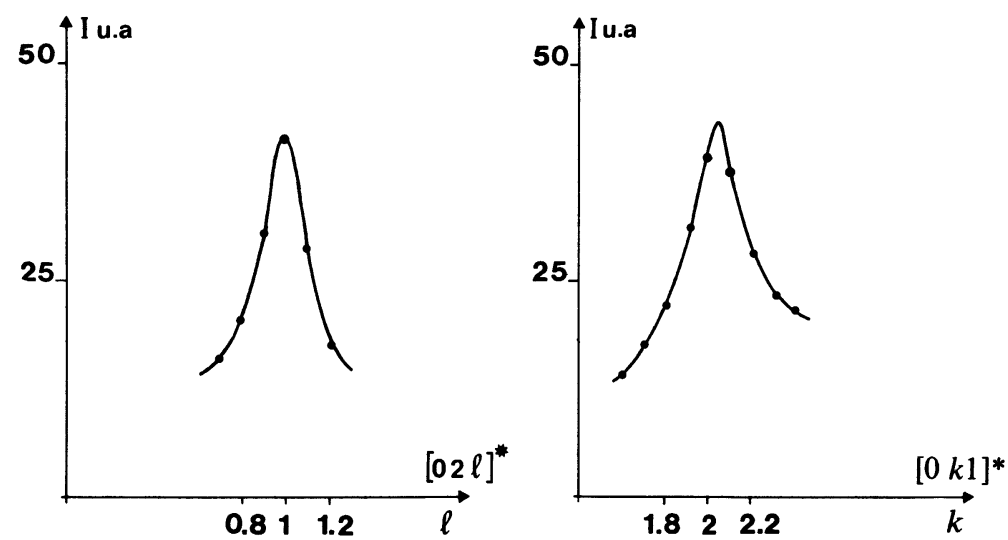

Fig. 5. - (a) Q scans along $\left[h 2^{21}\right]^{*}$ in the plastic phase (I) and in the glassy state $(\mathrm{Ig})$. In this later case, three diffuse spots are observed at the following $X$ points : 021, 121, 221 (location shown on Fig. 7a). (b) $\mathbf{Q}$ scans of the particular 021 spot along two perpendicular paths : $[02 l]^{*}$ and $[0 k 1]^{*}$.

being done along the perpendicular paths $[02 /]^{*}$ and $[0 k 1]^{*}$. This spot appears almost isotropical though it is slightly narrower in the $[02 l]^{*}$ direction.

The presence of diffuse spots at the $\mathrm{X}$ boundary points of the B.Z. shows that the local symmetry is lower than the average one due to the local disappearance of the $\left(\frac{\mathbf{a}+\mathbf{b}}{2}\right)$ type translation of the f.c.c. lattice. In the glassy phase, the orientational disorder of the molecular dipoles is quenched or remains as dynamic disorder with very long correlation times $(\tau>1000 \mathrm{~s})$. The diffuse zone boundary spots are associated with the existence of a short range order according to which the molecular dipoles are locally correlated in an antiparallel way. This is consistent with the very low value of the dielectric Kirckwood factor $\left(g_{\mathrm{K}} \approx 0.15\right)$ found when quenching the sample [8]. As the growth of a simple antiferroelectric ordering is incompatible with the f.c.c. structure and as the spots are reasonably sharp, we propose to describe the modification of the local order in the 
glassy crystal phase by assuming the existence of geared antiferroelectric clusters. If we assume an Ornstein-Zernike type correlation $\langle\mu(0) \mu(\mathbf{r})\rangle=\mathrm{e}^{-\boldsymbol{\xi} \mathbf{r}} /|\mathbf{r}|$ which makes the static susceptibility a Lorentzian : $\chi_{q_{x}}=\frac{K}{\xi_{x}^{-2}+q_{x}^{2}}$ (the $\mathbf{q}$ vector is taken with origin at the $\mathrm{X}$ point), the coherence length corresponding to the measured HWHM is : $\xi \sim 2 a \sim 20 \AA$.

The localization of the spots specifically at the $X$ points is consistent with the existence of fluctuation domains each of which corresponds to a sequence of opposite ferroelectric planes as shown on figure 6. Depending on the relative orientation of the dipolar axis with regard to these planes, two types of local arrangements compatible with the average structure are possible. In type (1) arrangement, the dipoles align in the ferroelectric planes. This gives an orthorhombic local structure. In type (2) arrangement one fourfold axis, lying along the dipoles, is preserved. This type (2) arrangement seems to be the most likely arrangement because it locally keeps one of the long range characters of the ordered monoclinic phase (II) which is the stable one at the same temperature : the $\mathrm{C} \equiv \mathrm{N}$ groups are locked inside the antiferroelectric plane. This gives a local tetragonal structure which has a cell twice as large as the primitive cubic cell. In each local cell, there are molecules with two opposite orientations (modulo $\pi$ ). The totality of the spots is divided into three groups associated with the three possible orientations of the local cell (Fig. $7 \mathrm{~b}$ ).

\section{Conclusion.}

We have shown the existence of strong intermolecular correlation effects in the glassy crystal phase of CN-ADM. We have suggested that this local order can be described by means of geared antiferroelectric fluctuation domains.

At present we are undertaking a more detailed study of the diffuse scattering. We have in mind to answer the following particular questions about local order.
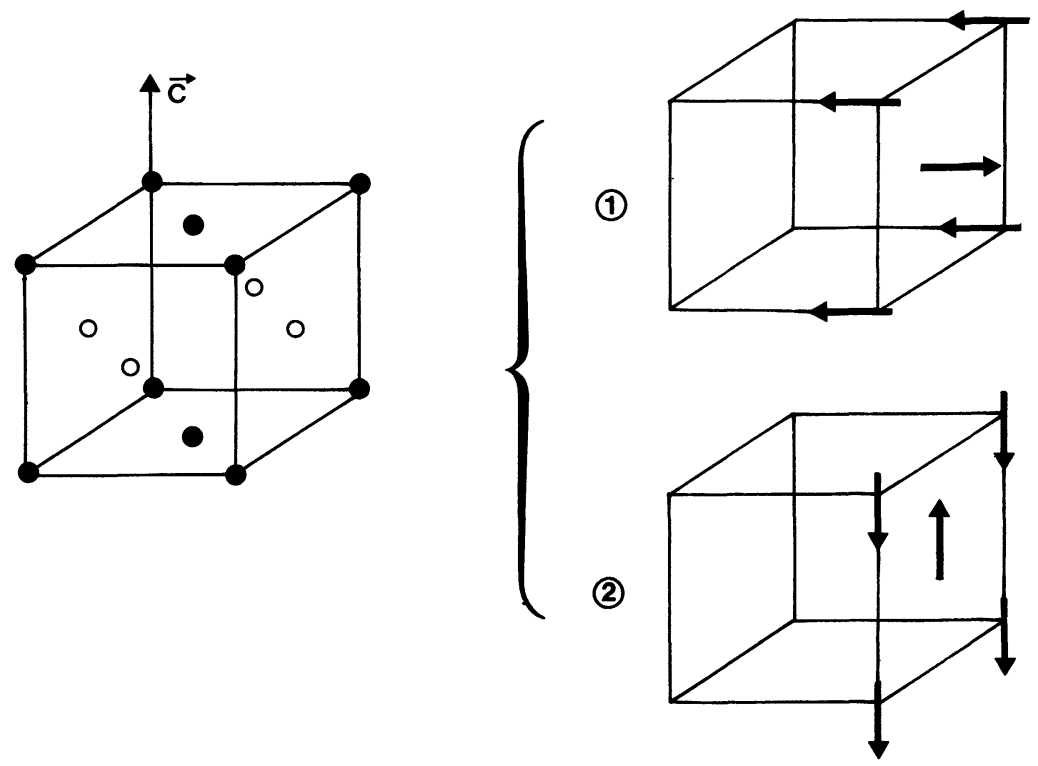

Fig. 6. - The two possible local antiparallel arrangements of the molecular dipoles corresponding to one "domain " in which opposite ferroelectric planes alternate along the c direction : (1) orthorhombic local structure; (2) tetragonal local structure. 
a)
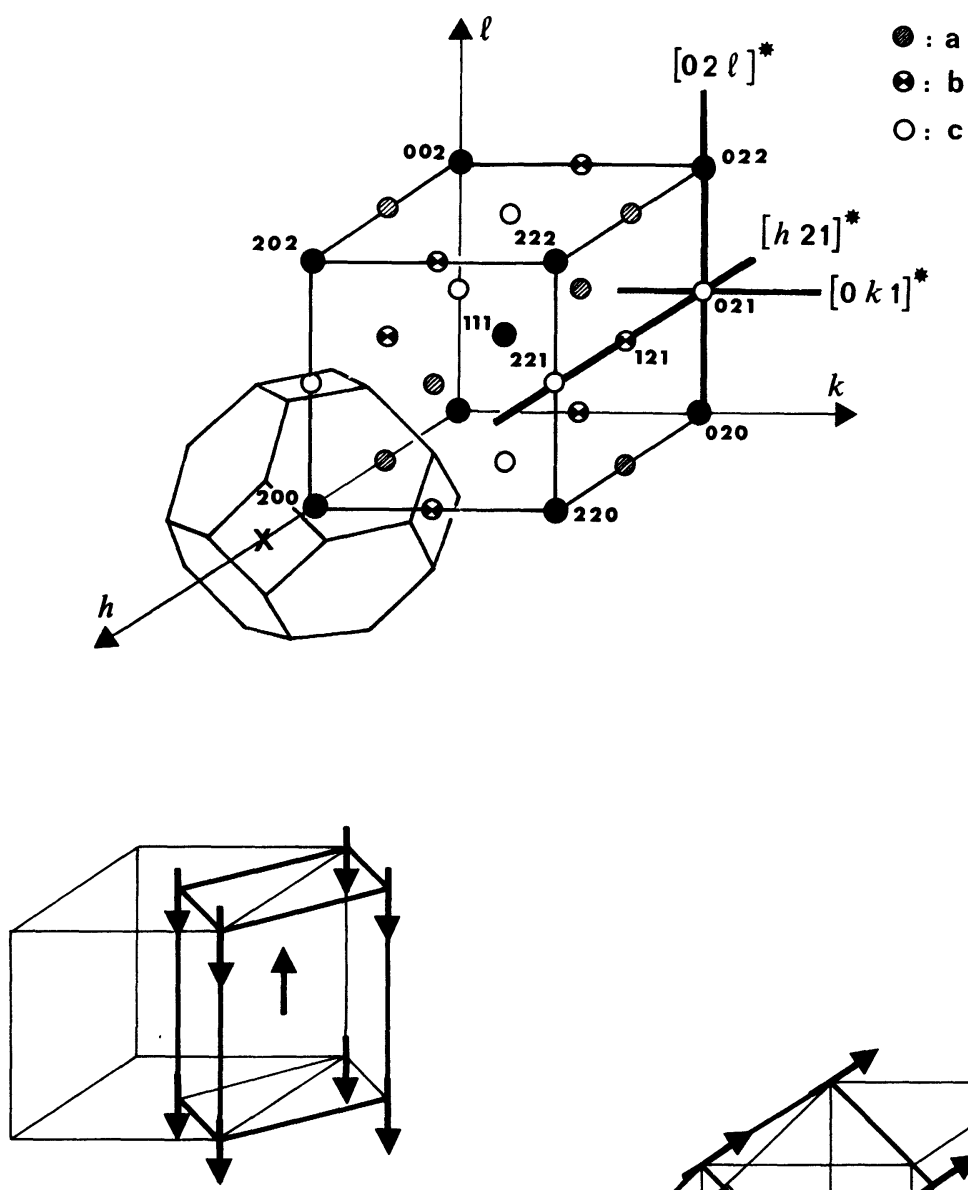

(c)
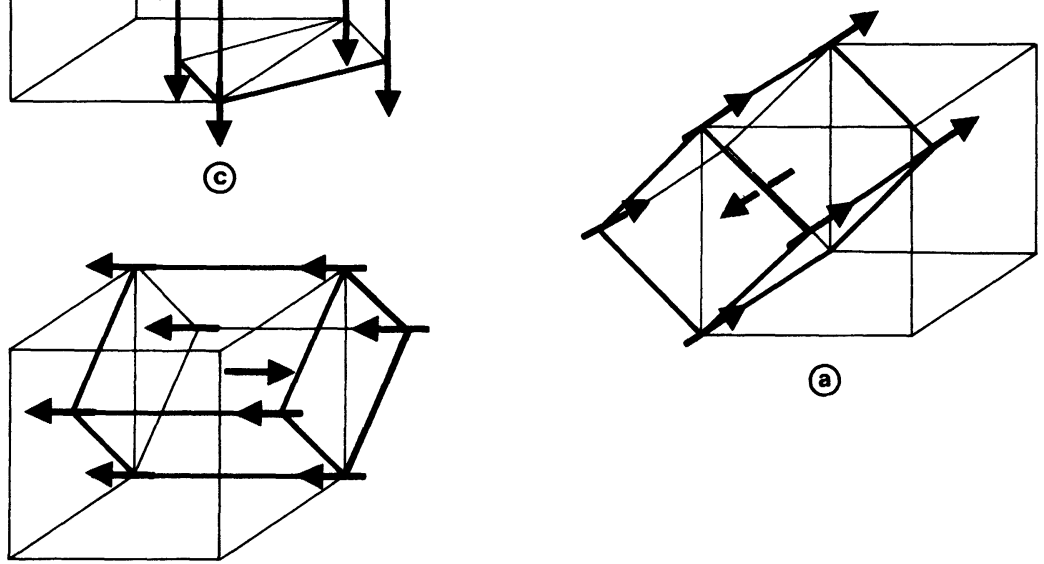

a)

b)

(b)

Fig. 7. - (a) Reciprocal space of the glassy state showing the observed zone boundary spots (X points). The separation into three types $(\mathrm{a}, \mathrm{b}$ and $\mathrm{c}$ ) corresponds to the hypothesis of a local tetragonal structure. (b) The corresponding orientations of the local tetragonal cell ( $a, b$ and $c$ ). 
- Is it the result of a clear change at $T_{\mathrm{g}}$ ?

We have succeeded in taking a photograph of the metastable phase $\left(\mathrm{I}^{\prime}\right)$ at $250 \mathrm{~K}$. It shows only a slight modification with regard to the room temperature scattering. This behaviour differs from the cyclohexanol case [6] in which " the diffuse scattering distinguishly drops when the temperature is lowered " in the plastic phase " and does not seem to decrease any more " when going to the glassy state.

- How is it modified by an annealing below $T_{\mathrm{g}}$ ?

We are conscious that the present slow cooling involves a partial annealing.

- Is the absence of scattering along the twofold axis and at the 300 point characteristic of its structure?

We hope that the presence of clearly marked intermolecular correlations will help to establish a more precise theoretical model of the glassy transitions.

\section{Acknowledgments.}

We are very grateful to $M$. Muller for growing very good single crystals, J. Carpentier for providing us photographic reproductions and Dr. W. Briels for reading the manuscript.

This research was, in part, supported by the DRET.

\section{References}

[1] Foulon, M., Amoureux, J. P., Sauvajol, J. L., Lefebvre, J., Descamps, M., J. Phys. C. 16(1983) L 265.

[2] Amoureux, J. P., Sauvajol, J. L., Bee, M., Acta Crystall., Sect. A 37 (1981) 97.

[3] Amoureux, J. P., Castelain, M., Bee, M., Arnaud, B., Shouteeten, M. L., Mol. Phys. 42 (1981) 119.

[4] Foulon, M., Amoureux, J. P., Sauvajol, J. L., Cavrot, J. P., Muller, M., to be published.

[5] Adachi, K., Suga, H., Seki, S., Bull. Chem. Soc. Jpn 41 (1968) 1073.

[6] Ceccaldi, D., Denoyer, F., Lambert, M., Szwarc, H., J. Physique Lett. 41 (1980) L-365.

[7] Foulon, M., AmoureuX, J. P., Lefebvre, J., to be published.

[8] Amoureux, J. P., Noyel, G., Foulon, M., Bee, M., Jorat, L., Mol. Phys., in publication (1984).

[9] Descamps, M., J. Phys. C. 15 (1982) 7265.

Sauvajol, J. L., Lefebvre, J., AmoureuX, J. P., Bee, M., J. Phys. C. 15 (1982) 6523.

[10] SaUvajol, J. L., LefebVRe, J., private communication. 